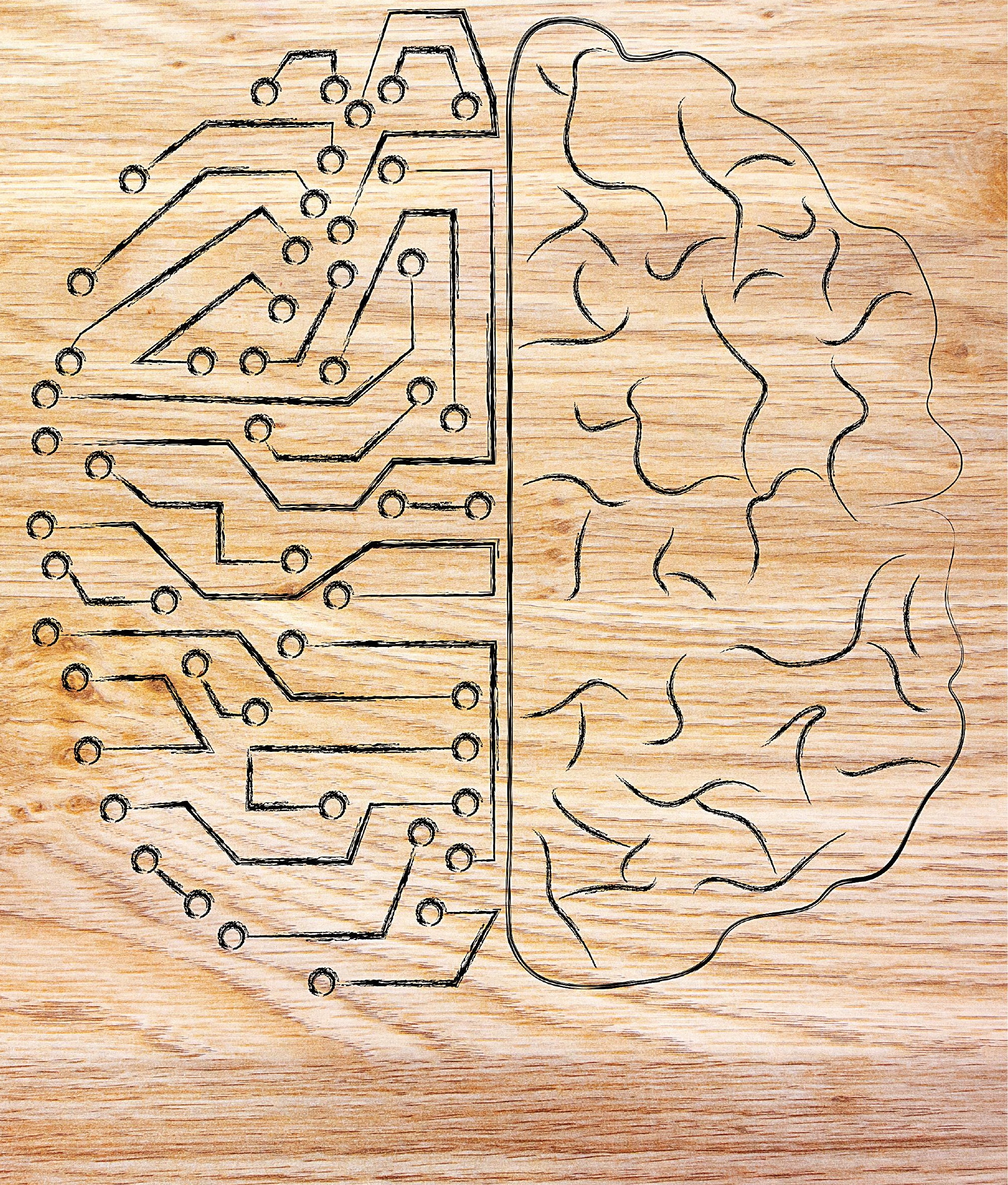




\section{SPECIAL TOPIC}

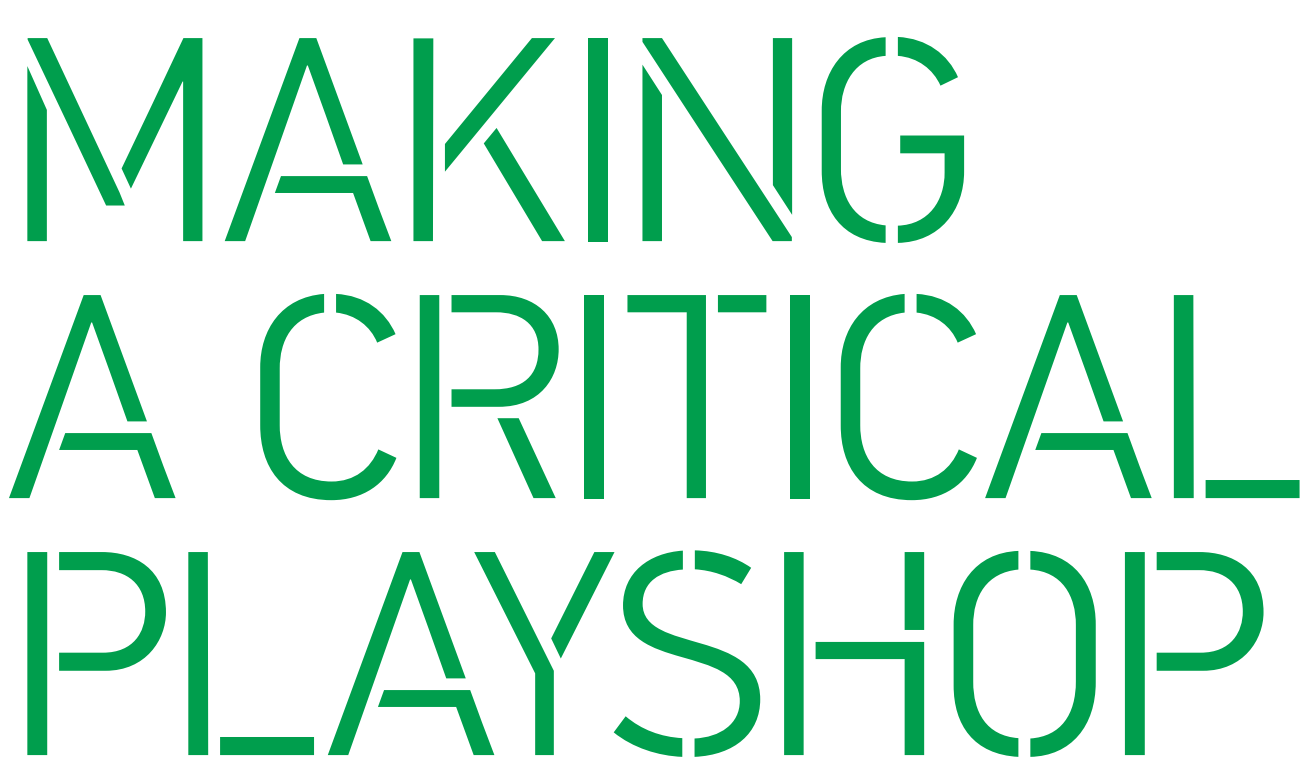

Austin L. Toombs, Open Lab at Newcastle University

Gabriele Ferri, Amsterdam University of Applied Sciences

Shannon Grimme, Indiana University Bloomington

Shad Gross, Angie's List

Michael D. Stallings, Blackbaud

Jeffrey Bardzell, Indiana University Bloomington

Shaowen Bardzell, Indiana University Bloomington

Criticality joined with making critical making - is a provocative topic in interaction design $[1,2,3]$. During our three-year ethnography of a small Midwestern hackerspace [4], we observed participants who might be described as already on the edges of critical making in their own practice, though they had not heard the term. We introduced the concept to some of them one evening in a conversation; it sparked their interest, and on a lark, critical playshops were born.

Critical playshops combine practices of hacking, academic theories of criticality and critical making, the event structures of a hackathon, and (at least in our initial intentions) the joyful open-endedness of play. Over the next few months, those of us on the research team, along with several hackers, planned a two-day event on the future of surveillance technologies. The idea was to use making and hacking to critically interrogate this topic. The term playshop is intended to communicate the participatory and constructive nature of a workshop but infused with a spirit of play - not only for the participants but also for us researchers. At the time, most of us were attending a graduate-level participatory design course (taught by Shaowen Bardzell) and were eager to set aside our usual academic methods and throw ourselves into something like a collectively enacted thought experiment-emergent, a bit out of our control, a little playful.

What follows is neither a proper scientific study nor a proper design study. It is, rather, a reflective account of what we attempted and how it came off, with some insights on why critical making turns out to be hard to do- even for veteran makers who think critically about technology.

\section{PLAYSHOP SETUP AND GOALS}

Though set in the hackerspace, the event targeted hackerspace members and the general public alike. People who wished to help design the event attended a separate four-hour workshop roughly a month prior. It was then that we coined the term playshop to foreground idea exploration and playful tinkering, while also hoping to avoid incorrectly signaling an educational focus (e.g., a workshop) that would have cast members in mentorship roles. In other words, the intention was to enable them to just play, rather than facilitate. In fact, many lamented that they, as a group, rarely have the opportunity to socially and playfully collaborate with other members in a structured context, due 


\section{SPECIAL TOPIC}

to the logistical difficulty of organizing such efforts.

This ability to play and collaborate was not only something members hoped to get out of co-organizing this event, but also something they wanted to model for newcomers in order to entice them to become members. To do so, the event needed to engage an audience with a wide variety of technological expertise without obligating members into assistant roles. For this reason, we collectively selected materials that are introductory in nature. The topic of surveillance was similarly motivated, as participating in speculations about its future can be a relatively easy way to approach academic theories of criticality.

\section{REFLECTIONS}

The design of this playshop mirrored in many ways the frameworks often used in the design of hackathons. We never explicitly questioned or resisted these hackathon tropes - such as the competition framing, the pressure to create a minimum viable product, and the assumption that everyone would participate similarly - and we did not realize until we were in the moment of the event that they were limiting our participants' abilities to engage with the prompt and materials in the playful ways we had expected.

The unnecessary, implicit competition framing. Throughout the process of designing the playshop, both the research team and our codesigners assumed the nature of the event as a kind of competition. Since most hackathons we had encountered included an extrinsic reward system for participating, we never considered whether our event could be valuable without awards for the best, most innovative, or most creative projects. In retrospect, whereas we intended play as genuinely open-ended, we realize that we (unintentionally) implied a more agonistic, game-like framing where people play to win.

The idea that the playshop could somehow be won caused at least two issues. First, assigning prizes required certain logistics (e.g., negotiating voting methods or attempting to create a rubric of sorts) that got in the way of the collaboration. Indeed, we quickly realized during the event that participants cared much more about playfully collaborating with their peers than about competing, as they frequently helped members of other teams or fluidly switched teams altogether.

Second, several participants expressed anxiety about importing ideas they had seen previous to the playshop, such as the idea to create a locket that blinked along with the wearer's heartbeat, which one participant had recently seen on instructables. These participants were worried that it might seem like they were cheating because their ideas either were not theirs or were not new ideas crafted specifically for this playshop. While we were perfectly willing to allow participants to import and modify ideas, we understand now that this anxiety was also likely linked to the competition framing.

The pressure to create led to limited experimentation. Included in this assumed competition framing, though a source of anxiety on its own, was the pressure to create a finished project. As time was short, participants stuck to materials, practices, and ideas they were familiar with to make sure they would not end the event with something halffinished. Even participants who chose new materials at the start of the event, such as the variety of conductive fabrics we supplied, slowly transitioned to more familiar materials as the deadline approached. Additionally, the ideas behind many participants' projects had fairly transparent genealogies: They were either ideas participants brought with them, such as an in-car police surveillance system, or they were inspired by something in the environment, such as a shirt to detect the creepiness of a hug that one group built because they knew a member of the

\section{We quickly realized during the event that participants cared much more about playfully collaborating with their" peer's than about competing.}

research team participated in the free hugs movement. In other words, they did not represent the kind of deep or critical thought process we had hoped to elicit.

One lesson from this is that it may not be reasonable to expect participants to have deep, creative insights about a theoretical topic while dealing with the practical challenges of working with unfamiliar tools and materials, especially if they are going to be judged based on the quality of their creations. Creativity often requires pushing the boundaries of what something can do or how something can be seen, even if pushing the boundaries is exploring how simple something can be. To be truly creative with a material (material in the broad sense, including new gadgets), it might be that one must already be deeply familiar with that material's boundaries and limitations.

It might have helped participants overcome the immediate reaction to stick with what they knew if we had scaffolded the materials differently for participants of various skill levels. As one participant noted, "Beginners should get to play with the nice, expensive stuff. Because in art, experts can do a lot more with less." Those who were more familiar with these forms of making and hacking could have been given lower-level materials and tools to work with, while those with less experience could have stuck with the composite materials that more effectively scaffolded the activities.

In reflecting more about the nature of play, this indeed seems to be something that productively resonates with some elements of game design: Players may want to choose the level of resistance that a system opposes, and experts may find it pleasantly challenging to have limited resources to play with. Several of our more experienced participants said they wished they had spent more time using materials new to them. If our playshop environment had emphasized open play over competing, perhaps it could have better provided the excuse these participants needed to practice with these new materials without having to justify spending time on them.

A multiplicity of participation/ play modalities. A variety of surprising participation modalities emerged throughout the event, and while we might not have initially wanted to design supports for these, we see now how they were valuable forms of play 

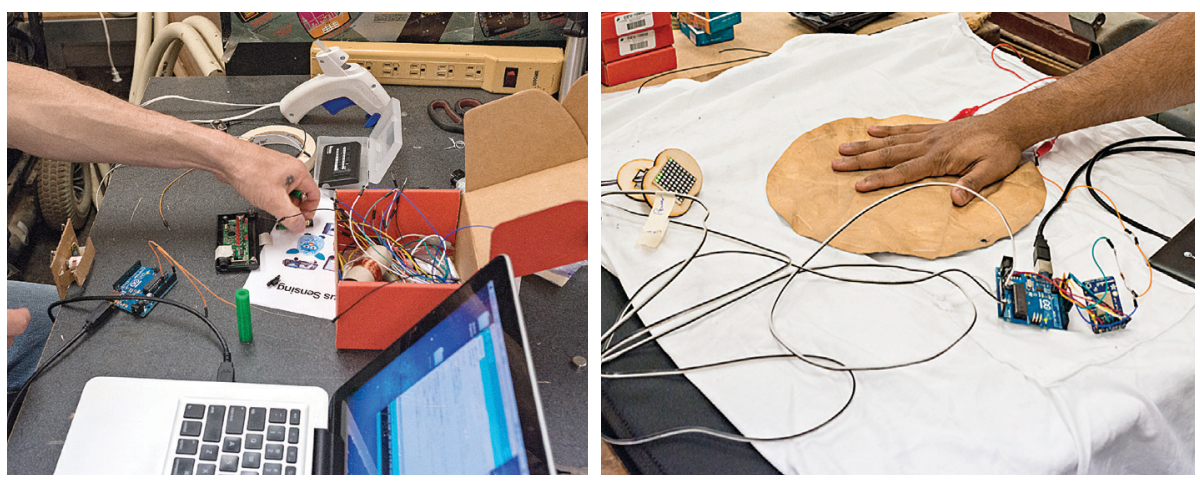

for these participants. One participant who showed up unexpectedly on the second day, for example, claimed to have a lot of thoughts about surveillance but was hesitant to try to make anything on his own. Rather than join an existing group mid-project, this participant chose to lead a speculative discussion group about the future of surveillance technologies.

While this was not a participation mode we had anticipated, he and those who joined him were content with what they were able to accomplish through their discussion. This unexpected effect of our attempts to facilitate emergent open play throughout the event suggests that playful and speculative discussions are one form of participation we may have to include in future playshop designs.

\section{FUTURE QUESTIONS}

There were surprising and unexpected elements in the process of running the playshop, and it was largely considered a success by participants, who appreciated the chance to work together in unique ways: Everybody loves a party, but not everybody likes to plan the logistics of it. In hindsight, having some flexibility toward emergent aspects of the eventand maintaining the ability to adapt to and generate knowledge from these changes - was both one of the most challenging parts of the playshop and one that allowed for some of the greatest levels of participation.

We have briefly outlined the main characteristics of critical playshops, as well as some practical insights, in the hope of expanding this collectively enacted thought experiment outside of our hackerspace. The design, organization, and implementation of this event have helped us solidify a few questions we did not initially realize we had about this research space. If we expressly define the act of making as playful and critical, which assumptions and behaviors should be supported and which conflict with these goals? How can emergent forms of play not necessarily designed for still be handled organically and inclusively in the event? How do participants themselves relate to playfulness and criticality, and what does it mean for us researchers to be playful? If the act of speculation, moving beyond a given framing to imagine what-ifs, can be considered playful, how can we better leverage play in designing events that hope to address speculative questions?

\section{ACKNOWLEDGMENTS}

We thank all of our participants, as well as Gillian Smith and Josh Tanenbaum, for comments on drafts. This work was supported in part by the National Science Foundation under awards 1002772 and 1513604 and the Intel Science and Technology Center for Social Computing.

\section{ENDNOTES}

1. Hertz, G. Making critical making: The value of DIY production. Proc. of the SIGCHI Conference on Human Factors in Computing Systems. ACM, New York, 2013.

2. Ratto, M. Critical making: Conceptual and material studies in technology and social life. The Information Societ 27, 4 (July 2011), 252-260. DOI: $10.1080 / 01972243.2011 .583819$

3. Grimme, S., Bardzell, J., and Bardzell, S. 'We've conquered dark:' Shedding light on empowerment in critical making. Proc. of the 8th Nordic Conference on Human-Computer Interaction: Fun, Fast, Foundational. ACM, New York, 2014, 431440. DOI: $10.1145 / 2639189.2641204$

4. Toombs, A.L., Bardzell, S., and Bardzell, J. The proper care and feeding of hackerspaces: Care ethics and cultures of making. Proc. of the 33rd annual ACM Conference on Human Factors in Computing Systems. ACM, New York, 2015. DOI: $10.1145 / 2702123.2702522$

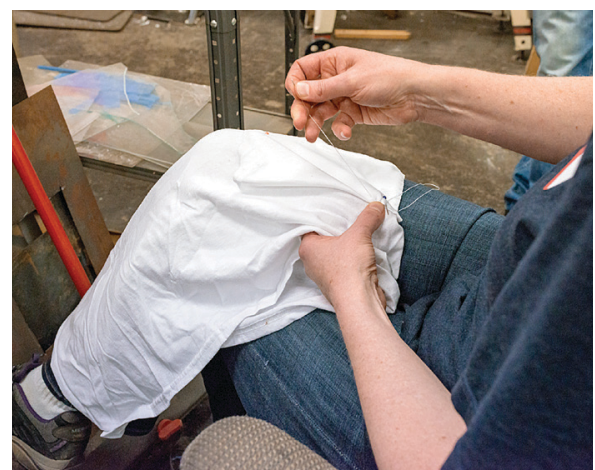

(4) Austin L. Toombs is a research associate at Open Lab, Newcastle University. He studies the impact that digital technologies have on how identities, interpersonal relationships, and communities develop and are maintained, with a focus on DIY, hacker, and maker contexts. $\rightarrow$ austin.toombsanewcastle.ac.uk

Gabriele Ferri is a postdoc researcher at the Amsterdam University of Applied Science. He was previously employed at Indiana University Bloomington. Combining his interests in playful and ubiquitous interactions, design futuring, and location-based

experiences, he pursues a research agenda focusing on the use of urban games as design tools to empower minorities. $\rightarrow$ g.ferriahva.nl

(d) Shannon Grimme lwww.shannongrimme coml is a tech and media producer, researcher, and writer. She will be receiving her master's in social informatics this year.

$\rightarrow$ seschenclaindiana.edu

Shad Gross received his Ph.D. from Indiana University's School of Information and Computing. His research has examined materiality within tangible interactions, DIY digital creativity support tools, and user experience design. He currently focuses on service design as a senior user experience design strategy researcher for Angie's List. $\rightarrow$ shadgrossagmail.com

(b) Michael D. Stallings is a user experience designer at Blackbaud. He designs for the K12 education space. Previous research foci have included critical design and design education. $\rightarrow$ contactamichaelstallings.me

(4) Jeffrey Bardzell is an associate professor of $\mathrm{HCl} /$ design in the School of Informatics and Computing at Indiana University Bloomington. His research foci include research through design, user experience and aesthetics, and digital creativity, with particular emphasis on critical design and design criticism.

$\rightarrow$ jbardzelaindiana.edu

(1) Shaowen Bardzell is an associate professor of informatics at Indiana University's School of Informatics and Computing. Recent research foci have included criticality in design, care ethics and feminist utopian perspectives on IT, and culture and creative industries in Asia. $\rightarrow$ seludindiana.edu 